\title{
Feasibility Study on Structure Optimization of Filter Elements for Hydraulic Pipelines
}

\author{
Liwei Zhao ${ }^{1,}$, , Ying Wang ${ }^{2, b}$ \\ ${ }^{1}$ School of Mechanical Engineering, Shenyang Ligong University, Shenyang 110159, China \\ ${ }^{2}$ Wuxi Risho Technology Co., Ltd. Wuxi 214000, China \\ azhaoliwei33@126.com, ${ }^{\mathrm{b}}$ YING.KING@hotmail.com
}

Keywords: filter element, filtration Resistance, numerical simulation, CFD, flow field analysis

\begin{abstract}
The goal of this paper is to form a mathematic model indicating the relationship between the structural parameters of a filter element and its pressure drop. Under study a plait stainless steel filter element used for a hydraulic pipeline filtration system, search for the optimized structural parameters of a filter element used for XYZ-100 lubricating stations and simulate numerically its optimized structural parameters that are worked out theoretically by means of the FLUENT technology of CFD software. Based on the theoretical model, the optimized structural parameters are proved to be feasible through contrasting the theoretical outcome with the simulated outcome.
\end{abstract}

\section{Introduction}

A filter element is the core element for a hydraulic pipeline filtration system. The past twenty years witnessed the growing development of the technology concerning filter elements for hydraulic pipelines, and remarkable progress in theory has been achieved by researchers at both home and abroad [1], for example, Feng Chaoyang et al conducted experimental researches on the relationship between the structural parameters of a nuclear-grade air filter and the resistance that it suffers, and achieved some results.

Based on the theoretical base for plait filter elements, this paper deals with the relationship between the structural parameters of a filter element and the resistance that it suffers, and aims to make suggestions for the production of filters and type selection of filter products through the numerical simulation test on the resistance against a plait filter element for hydraulic pipelines.

\section{Theoretical model of resistance against a filter element}

2.1 Formation of the model. Based on the structure of plait filter elements, a model is formed as shown in Fig. 1, and the structural parameters are as shown in Figs 1 and 2, where $h$ is the plait height, $\mathrm{N}$ is the number of plaits as, the height of the filter element as $\mathrm{H}$, and the external and internal diameters of the filter element plait as $\mathrm{D}$ and $\mathrm{d}$.
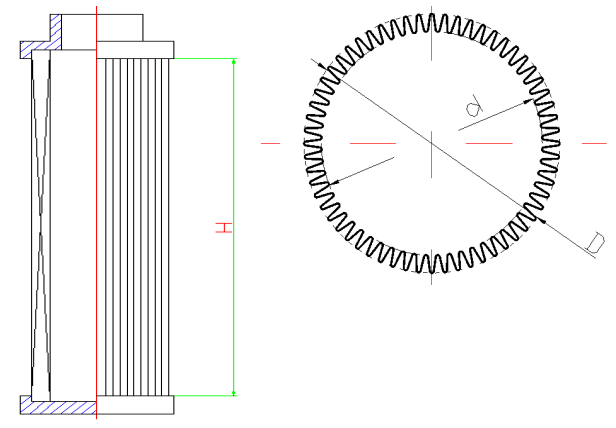

Fig.1 Filter element model

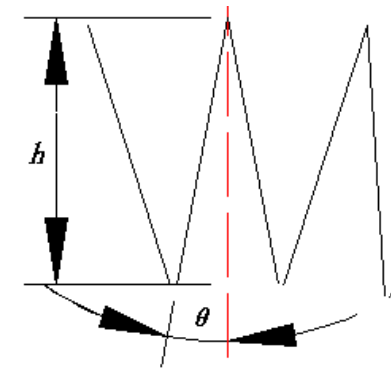

Fig.2 Structural parameter of plait 


\subsection{Analysis of the resistance against the filtering surface of a filter element}

(1) Theoretical model of the filtering surface of a filter element

The filtering surface of a filter element can be defined as a porous medium model. Under the condition of low flow velocity and small Reynolds number, the resistance at both ends of the porous medium is distributed in line with Darcy's Law:

$$
\frac{\Delta P}{L}=\frac{\mu}{K} V
$$

where $\Delta P$ represents the pressure $\operatorname{drop}(P a)$;

$L$ represents the thickness of the porous medium $(m)$;

$\mu$ represents the dynamic viscosity of fluid $(\mathrm{Pa} \cdot \mathrm{s})$;

$K$ represents the permeability $\left(m^{2}\right)$;

$V$ represents the mean flow velocity of fluid $(\mathrm{m} / \mathrm{s})$.

Permeability $K[2]$ :

$$
K=\frac{d_{f}^{2}}{f(\alpha)}
$$

where: $f(\alpha)$, a dimensionless force suggested by Davies[3], is the function of fill rate $\alpha$, which can be calculated through the following empirical formula[4]:

$$
f(\alpha)=2.653 \alpha+39.34 \alpha^{2}+144.5 \alpha^{3}
$$

where $d_{f}$ is the diameter of fiber silk .

(2) Calculation of Reynolds number

Supposing that the velocity of flow through the filter element is $V$, then the Reynolds number can be calculated by means of Formula (4):

$$
\operatorname{Re}=\frac{V \cdot d_{f}}{v}
$$

where $v$ represents the kinematic viscosity.

(3) Pressure loss caused by the filtering surface $\Delta P m$

The pressure loss of a fluid when flowing across the filtering surface of the filter element can be calculated as follows[5]:

$$
\Delta P_{m}=\frac{1}{2} \cdot \xi \cdot\left(\frac{Q}{A_{0}}\right)^{2} \cdot \rho
$$

where $\Delta P_{m}$ represents the pressure drop of the filtering surface of the filter element $(P a)$;

$Q$ represents the flow velocity $\left(\mathrm{m}^{3} / \mathrm{s}\right)$;

$A_{0}$ represents the through-hole area of the filter screen $\left(\mathrm{m}^{2}\right)$;

$\rho$ represents the fluid density $\left(\mathrm{kg} / \mathrm{m}^{3}\right)$;

$\xi$ represents the resistance coefficient.

where the resistance coefficient can be calculated as in Table 1.

\subsection{Pressure loss caused by the plait structure $\Delta P g$}

Based on the theoretical study made by Fu Haiming et al[6] on plait filters, we can work out the pressure loss $\Delta P_{g}$ caused by the plait structure as follows:

$$
\Delta P_{g}=\left|\Delta P_{g}^{\prime}\right| \cdot \rho V^{2}
$$




$$
\begin{aligned}
& \Delta P_{g}^{\prime}=\frac{\mu}{\rho V} \cdot \frac{\cos ^{2} \theta \cdot \sin \theta}{L} \cdot\left[1-\left(\frac{L}{h \cdot \sin \theta}\right)^{3}\right]+ \\
& \frac{3 \mu}{\rho V} \cdot \frac{\cos ^{2} \theta}{\tan ^{2} \theta} \cdot \frac{\sin \theta}{L} \cdot\left[1-\left(\frac{L}{h \cdot \sin \theta}\right)\right]+ \\
& \frac{1}{2} \cos ^{2} \theta \cdot\left(1+\frac{1}{2} \cos ^{2} \theta\right) \cdot\left[1-\left(\frac{L}{h \cdot \sin \theta}\right)^{2}\right]+ \\
& \frac{1}{4} \cos ^{4} \theta \cdot\left[1-\left(\frac{L}{h \cdot \sin \theta}\right)^{3}\right]-\frac{9}{40} \cos ^{4} \theta \cdot\left[1-\left(\frac{L}{h \cdot \sin \theta}\right)^{4}\right]
\end{aligned}
$$

\subsection{Total pressure drop of the filter structure $\Delta P$}

According to Formulas (5) (7), we can find out:

$$
\Delta P=\Delta P_{m}+\Delta P_{g}
$$

Diminishing the plait angle and increasing the number of plaits and plait height will lead to increases in filtering area and flow $\operatorname{rate}(\theta, h)$ and a decrease in pressure drop, which is conducive to filtration. However, to seek only the maximum number of plaits in attainment of greater area will surely lead to a smaller plait span, which will, on the contrary, lead to a greater structural resistance. Therefore, there must be a pair of optimal values $(\theta, h)$, which makes the pressure loss $\Delta \mathrm{P}$ minimum.

Table 1 Calculation of $\xi$

\begin{tabular}{cc}
\hline $\mathrm{Re} \geq 400 \quad \xi=1.3 \cdot\left(1-\frac{A_{0}}{A}\right)+\left(\frac{A_{0}}{A}-1\right)^{2}$ \\
\hline $\mathrm{Re}<400$ & $\xi^{\prime}=\eta \cdot \xi$ \\
\hline
\end{tabular}

Table 2 Coefficient of correction affected by $R e$

\begin{tabular}{ccccccc}
\hline $\mathrm{Re}$ & 50 & 100 & 150 & 200 & 300 & 400 \\
\hline$\eta$ & 1.44 & 1.24 & 1.13 & 1.08 & 1.03 & 1.01 \\
\hline
\end{tabular}

Note: $A$ - filtering area, $m^{2} ; \eta$-Coefficient of correction affected by $R e$, see Table 2.

\section{Study of the Optimized Value of the Plait Structure of Filter Elements}

Putting relevant parameters into Formulas $(5) \sim(8)$ and finding out the results through operations, as shown in Fig.3, there must be the optimal value, which makes the pressure loss minimum, that is, there will be the minimum pressure loss. Therefore, we can ultimately find out the optimal value according to Formula (9), as shown in Fig. 4.

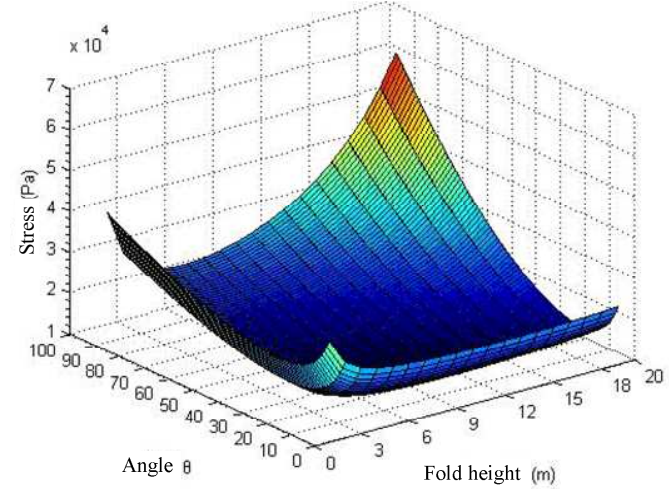

Fig.3 Surface diagram of the relationship between plait height, angle and pressure drop.

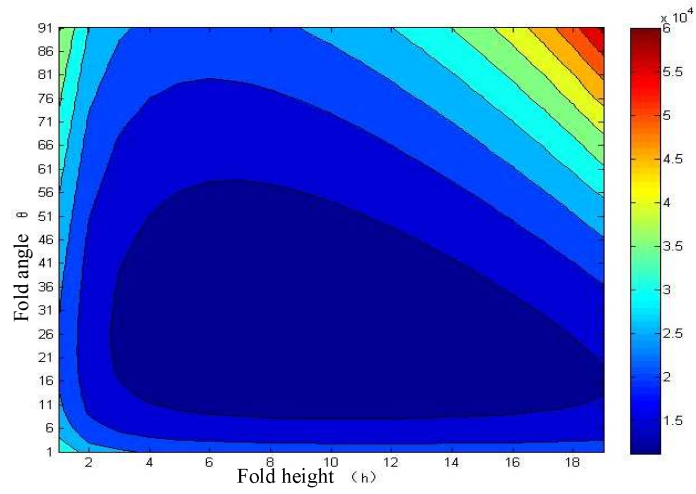

Fig.4 Pressure distribution diagram

The optimized values corresponding with the minimum pressure drop $\Delta \mathrm{P}=11201 \mathrm{~Pa}$ are:

Plait height: $h=10 \mathrm{~mm}$;

Plait angle: $2 \theta=7^{\circ}$; 
According to the geometrical relation of a plait structure, the number of plaits can be calculated as follows:

$$
N \approx \frac{\pi d}{2(h \cdot \tan \theta+L)}
$$

Substituting the above values for the $h$ and $\theta$ in Formula (9), we can find out that the optimized number of plaits of the filter element $N$ (round-off number) is 71 .

\section{CFD numerical Simulation Experiment}

With the limitation to the experimental conditions for studying the pressure loss of filter elements and increasing reliability of the computational fluid mechanics (CFD), more and more evidence for the practicability of CFD in the area of flow field is brought up [12]. Therefore, we can conduct simulate numerically the flow field of a filter element by means of the FLUENT of CFD software, conduct simulation experiments aiming at the flow field of a filter element, and verify the reliability of such theories according to the results of simulation experiments.

The following hypotheses are made in the simulation calculation:

(1) For the flow process, consideration is given only to the state of the fluid after it becomes stable, but not to the instantaneous state at the beginning and the end.

(2) No consideration is given to the effect of temperature during the filtration process, that is, the fluid is regarded as flowing at ambient temperature without heat transfer.

(3) Aiming only at the study of the distribution of velocity and pressure within the flow field, regardless of the effect of impurity.

(4) Ignoring the effect of gravity.

\subsection{Simulation calculation}

The model can be divided into 72134 grids, the detailed structural parameters and flow attribute of which are as shown in Article 2.1. The calculation can be done by means of the k- $\omega$ model, and the discrete equations can be solved by means of the semi-implicit method for pressure linked equations (SIMPLE algorithm). The residual criterions for the convergence of continuity are both set to $10^{-4}$.

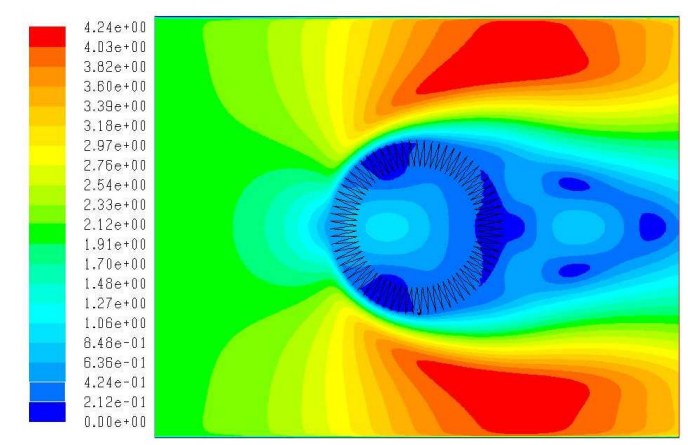

Fig.5 Cloud atlas of flow velocity distribution

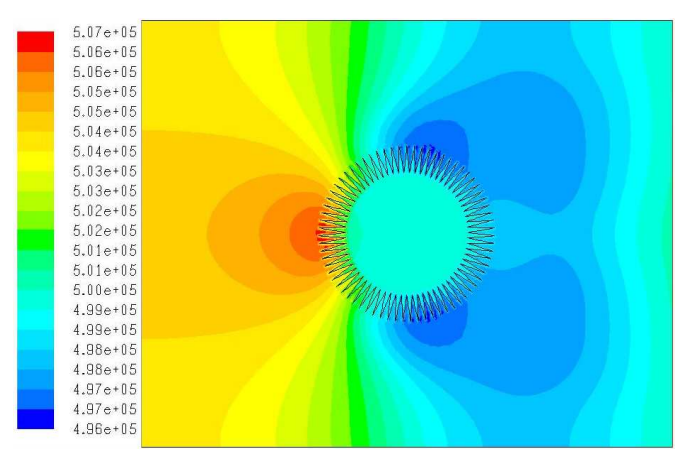

Fig. 6 Cloud atlas of pressure distribution

Through operations, we can obtain a cloud atlas of flow velocity distribution and a cloud atlas of pressure distribution, as shown in Figs. 5 and 6.

According to the cloud atlas of flow velocity distribution as shown in Fig.5, the initial flow velocity is $2.07 \mathrm{~m} / \mathrm{s}$. Because of the blocking effect of the filter element, a bypass flow is formed behind the filter element, at the same time, the flow velocity of the fluid that has flowed into the filter element declines to $0.5 \mathrm{~m} / \mathrm{s}$ or so. The velocity distribution of the flow field is as shown in Fig. 7 after processed with Tecplot software. On the other hand, according to Fig. 6, the pressure distributed before the filtering surface is about $506000 \mathrm{~Pa}$, while $497000 \mathrm{~Pa}$ or so after passing the plait filtering surface, distributed evenly inside the filter element, as shown in Fig. 8. 


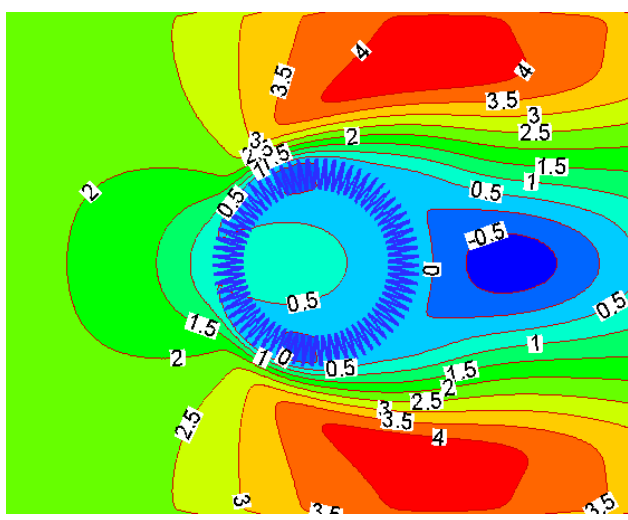

Fig. 7. Flow velocity distribution chart $(\mathrm{m} / \mathrm{s})$

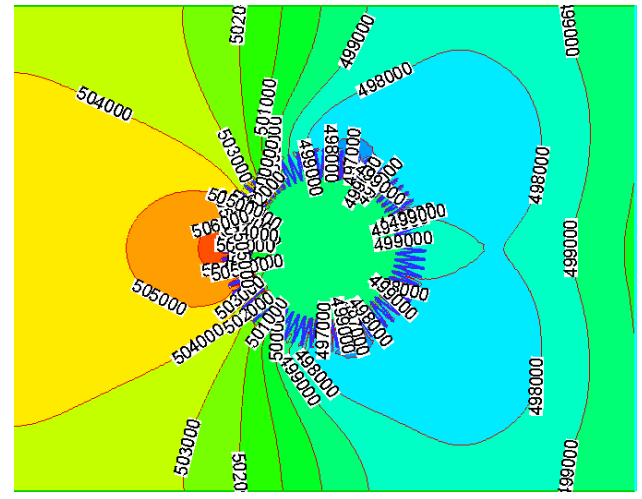

Fig. 8. Pressure distribution chart $(\mathrm{Pa})$

But the error of pressure loss calculated according to the optimization calculation result as described in article 1.2, contrasted with the computer numerical simulation result, is about $19.6 \%$.

Through analysis, the error is mainly caused by:

(1) The optimization calculation, which eliminates all the external influences including inter-disturbance between fluids, and that from pressure difference between flow fields, is completely the theoretical calculation mathematically; but the computer numerical simulation, which simulates the real flow state of a fluid through the finite element calculation, is closer to the actual movement state.

(2) In conducting an optimization calculation, the hypothesis that the fluid enters and leaves perpendicularly the filtering medium shortens the actual route through which airflow passes over the filtering medium. But in the simulation experiment, the fluid does not enter the filter element perpendicularly, furthermore, the flow fields between adjacent plaits may disturb each other, therefore, the pressure loss value calculated will deviate somewhat from the theoretical value.

For the above reasons, we say the error is inevitable. But the error is within $20 \%$, so we hold the opinion that the result calculated with the proposed theoretical calculation method is consistent with the simulated experiment result, and that, under the condition that the parameters of the filtering medium are given and the optimized parameters of the filter element structure can be worked out through optimization calculation, it is feasible to verify the correctness of an optimization scheme through computer numerical simulation experiments.

\section{Conclusion}

(1) According to the theoretical formula, the filter element resistance can be expressed as the function of such factors as the plait angle $\theta$ of the filter element structure and plait height $\mathrm{h}$, as well as the fluid density $\rho$, dynamic viscosity $\mu$ and parameters of the filtering surface, thus we put forward this mathematical model for structural optimization.

(2) Through simulating the characteristics of the flow field inside a filter element two-dimensional model with CFD method, we can obtain related data and cloud atlases of distributions of flow velocity and pressure, which basically reflect the real flow state of the fluid inside the filter, and make the flow state of the fluid inside the filter visible clearly. The numerically simulated experimental verification made with the CFD technology by virtue of the optimized structural parameters obtained through mathematical method not only saves the cost for practical experiments, but obtain an outcome more direct viewing than the practical experiment.

(3) By contrasting the structural parameters of a filter element obtained through the mathematical optimization calculation with that obtained from the numerical simulation experiment, and based on our experience in their applications in the practical engineering projects, we hold the opinion that the filter element structural parameter optimization model proposed in this paper is feasible. 


\section{References}

[1] Liu Danghua, Gao Yuan'an, Liu Yong. Design of the Test on the Filtering Performance of Filter Elements . Hydraulic and Pneumatic, 2009,(1):4-6.

[2] Cai Jie. Air Filters ABC. Beijing: China Architecture Industry Press, 2002.

[3] C.N Davis. Air Filtration. Beijing: Nuclear Energy Press, 1979.

[4] Rao, N, Faghri, M. Computer Modeling of Aerosol Filtration by Fibrous Filters. Aerosol Science and Technolosy, 1988,8(2):133-156.

[5] Dai Tianyi. Filters-Design, Manufacture and Use. Beijing: Chemical Industry Press, 2009.

[6] Fu Haiming, Xu Fang, Jin Ruifang. Relation between the filtering resistance of a Plait Aerosol Filter and its Filtering Parameters. Journal of Huaqiao University (Natural Science), 2010, 31(3): 307-312.

[7] Wang Ruijin. Introduction to Fluent Technology and Applications Beijing: Tsinghua University Press, 2007.

[8] Jiang Fan. Advanced Applications of Fluent and Case Analysis. Beijing: Tsinghua University Press, 2008.

[9] Zhang Fengchao. Numerical Simulation Study of Multi-pipe High Efficiency Air Filters. Shanghang: Donghua University, 2006. 